\title{
Model Order Reduction Zero-Pole Characterization and Computer-Aided Design of Microwave Circuits
}

\author{
Belén Carrasco and Valentín de la Rubia
}

\begin{abstract}
A reduced-order model is applied to finite element analysis of microwave circuits. The matrix-valued transfer function of the system is obtained, which is described as a scattering matrix transfer function. Due to the application of model order reduction techniques, the scattering matrix transfer function of the microwave circuit is detailed as a low dimension system, which allows us to efficiently obtain the frequency behavior of the system. In addition, the fundamental characteristics of this transfer function are discussed and a procedure to efficiently obtained the zelo-pole characteristics of the microwave filter is addressed. Finally, real-life applications will illustrate the capabilities of this approach.
\end{abstract}

Index Terms-Computer aided engineering, design automation, finite element methods, Galerkin method, microwave circuits, reduced basis methods, reduced-order systems.

\section{INTRODUCTION}

Increasing deployment of telecommunication services is urging microwave industry to speed up product development time, where not only prototyping and final tuning steps in the production line have to be reduced, but also simulation time to get an actual electrical design by means of computeraided design (CAD). A significant cost reduction can be achieved in prototyping and tuning steps if electrical designs are optimized to do so, in addition to satisfying electrical specifications. Performing robust electrical designs has thus become of paramount importance, and powerful numerical techniques capable of predicting electromagnetic behavior are at the heart of this robust design.

The Finite Element Method (FEM) is widely used in Microwave Engineering for its capability to predict the electromagnetic behavior in microwave circuits prior to construction. Due to its rather time-consuming characteristics, the FEM has been traditionally used in industry as an analysis tool, rather than a methodology for computer-aided design (CAD). However, current computational resources are leading to bruteforce design approaches, where global optimization techniques are taken into account. In this scenario, a large number of analyses are needed, and every single analysis may be timeconsuming. As a result, this optimization strategy can still be considered to be non-practical.

This work is organized as follows. In Section II we review the time-harmonic Maxwell's equations in variational form and state the global to local mode representation basis. Section III shows some numerical example and illustrates the capabilities of the proposed approach. Finally, in Section IV, we comment on the conclusions.

\section{PRoblem Statement}

Let us consider a two-port microwave circuit, namely, a microwave filter. The filter transfer function is represented by a scattering matrix transfer function [1]. After discretization of the time-harmonic Maxwell's equations by means of FEM, we get the following linear dynamical system representation of the microwave circuit:

$$
\left(\begin{array}{c}
b_{1} \\
b_{2} \\
0
\end{array}\right)=\left(\begin{array}{ccc}
0 & 0 & \beta_{1}^{T} \\
0 & 0 & \beta_{2}^{T} \\
s \beta_{1} & s \beta_{2} & \mathcal{S}+s \mathcal{U}+s^{2} \mathcal{T}
\end{array}\right)\left(\begin{array}{c}
a_{1} \\
a_{2} \\
x
\end{array}\right)
$$

where $\mathcal{S}$ and $\mathcal{T}$ are the stiffness and mass matrices, $\mathcal{U}$ is the port loading matrix, $\beta$ is the excitation vector, $x$ stands for the state-space variable and $s$ is the complex frequency. Once the state-space variable, which represents the FEM discretization of the electric field, is eliminated from equation (1), the scattering matrix transfer function arises, thus,

$$
\left(\begin{array}{l}
b_{1} \\
b_{2}
\end{array}\right)=-s\left(\begin{array}{c}
\beta_{1}^{T} \\
\beta_{2}^{T}
\end{array}\right)\left(\mathcal{S}+s \mathcal{U}+s^{2} \mathcal{T}\right)^{-1}\left(\begin{array}{ll}
\beta_{1} & \beta_{2}
\end{array}\right)\left(\begin{array}{l}
a_{1} \\
a_{2}
\end{array}\right) .
$$

It should be noted that the dynamical system (1) has a rather large state-space, thus solving this system frequency by frequency cannot be afforded. In this regard, we use MORe to carry out a fast frequency sweep. The goal in MORe is to identify the manifold, or reduced-basis space, in which the actual dynamics of system (1) take place in an efficient way [2], [3], [4], [5], [6], [7], [8]. As a result of this MORe process, we obtain a scattering transfer function which is really easy to evaluate due to its low dimension, thus,

$$
\begin{aligned}
& \left(\begin{array}{l}
b_{1} \\
b_{2}
\end{array}\right)=-s\left(\begin{array}{c}
\tilde{\beta}_{1}^{T} \\
\tilde{\beta}_{2}^{T}
\end{array}\right)\left(\tilde{\mathcal{S}}+s \tilde{\mathcal{U}}+s^{2} \tilde{\mathcal{T}}\right)^{-1}\left(\begin{array}{ll}
\tilde{\beta}_{1} & \tilde{\beta}_{2}
\end{array}\right)\left(\begin{array}{l}
a_{1} \\
a_{2}
\end{array}\right) \\
& \left(\begin{array}{l}
b_{1} \\
b_{2}
\end{array}\right)=S(s)\left(\begin{array}{l}
a_{1} \\
a_{2}
\end{array}\right) .
\end{aligned}
$$

The purpose in this work is to extract the fundamental characteristics of the microwave in the scattering matrix transfer function (3), namely, the poles and zeros of $S(s)$, in a straightforward way.

\section{A. Zero-Pole Characterization of the Reduced-Order Model Transfer Function}

The scattering matrix transfer function $S(s)$ in (3) is a meromorphic function in $\mathbb{C}$. The poles in this transfer function 


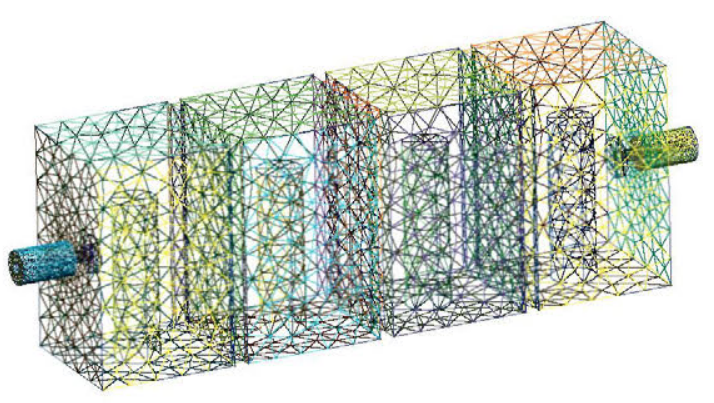

Fig. 1. $4^{\text {th }}$ order combline filter. Geometry description.

can be identified by solving a quadratic eigenvalue problem for the system in (3) $\tilde{\mathcal{S}}+s \tilde{\mathcal{U}}+s^{2} \tilde{\mathcal{T}}$, which can be further reduced to a linear eigenvalue problem using standard methods. However, the computation of the zeros in this matrix-valued transfer function is not straightforward for the general case. Here, we propose a methodology to deal with this problem which is based on the properties of meromorphic functions, namely, each pole in a meromorphic function is associated to a zero in the same meromorphic function which can be located either at infinity or at a finite position.

As a result of this property for meromorphic functions, once the location of the poles in the function is known, each zero associated to this meromorphic function can be tracked by means of any of the procedures discussed in [9], [10], [11], [12], [13]. Then, the zeros of this transfer function are found in an iterative way.

Once the zero-pole characterization of the microwave circuit is obtained, an optimization procedure can be followed to carry out computer-aided design for the microwave circuit taken into account [14].

During the presentation in the conference we will go through all the details and show several design examples to provide the advantages in this formulation.

\section{NUMERICAL RESULTS}

In this section, we apply the proposed methodology for the zero-pole characterization of a $4^{\text {th }}$ order combline filter. See Fig. 1, where the geometry of the combline filter is plotted.

The frequency response for this filter in shown in Fig. 2. In order to achieve this response, the zero-pole characteristics of the microwave filter have been extracted from the MORe procedure by means of the methodology described above. In addition, a zero-pole based optimization procedure [14] is carried out. The zero-pole diagram of this filter is detailed in Fig. 3, where the target zero-pole diagram for an equi-ripple response is shown as well with square points in the normalized low-pass domain.

\section{CONCLUSIONS}

A reduced-order model has been applied to finite element analysis of microwave circuits. The scattering matrix-valued transfer function of the system has been obtained. Due to the application of model order reduction techniques, the scattering

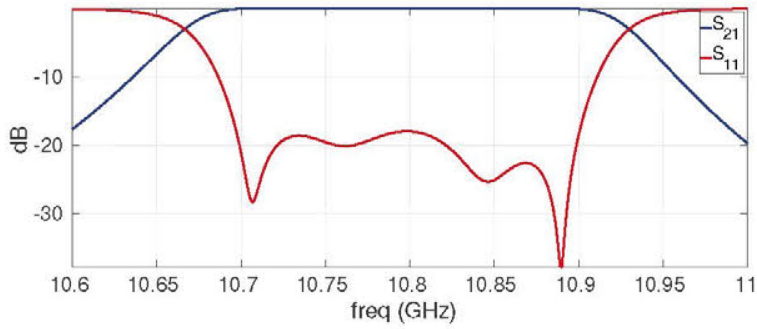

Fig. 2. $4^{\text {th }}$ order combline filter. S parameters response.

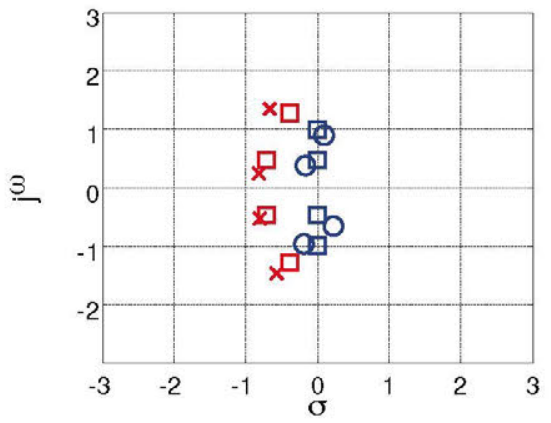

Fig. 3. $4^{\text {th }}$ order combline filter. Zero-pole characteristics in the normalized low-pass domain.

matrix transfer function of the microwave circuit has been detailed as a low dimension system, which allows us to efficiently obtain the frequency behavior of the system in the frequency band of interest. In addition, the fundamental characteristics of this transfer function have been discussed and a procedure to efficiently obtained the zelo-pole characteristics of the microwave filter has been addressed. A combline filter has been studied to show the applicability of the propose approach.

\section{REFERENCES}

[1] R. Baltes, A. Schultschik, O. Farle, and R. Dyczij-Edlinger, "A finiteelement-based fast frequency sweep framework including excitation by frequency-dependent waveguide mode patterns," IEEE Trans. Microwave Theory Tech., vol. 65, no. 7, pp. 2249-2260, Jul. 2017.

[2] A. Sommer, O. Farle, and R. Dyczij-Edlinger, "Certified dual-corrected radiation patterns of phased antenna arrays by offline-online order reduction of finite-element models," J. Comput. Phys., vol. 299, pp. $22-44,2015$.

[3] V. de la Rubia, U. Razafison, and Y. Maday, "Reliable fast frequency sweep for microwave devices via the reduced-basis method," IEEE Trans. Microwave Theory Tech., vol. 57, no. 12, pp. 2923-2937, Dec 2009.

[4] V. de la Rubia and M. Mrozowski, "A compact basis for reliable fast frequency sweep via the reduced-basis method," IEEE Trans. Microwave Theory Tech., vol. 66, no. 10, pp. 4367-4382, Oct. 2018.

[5] W. Wang, G. N. Paraschos, and M. N. Vouvakis, "Fast frequency sweep of FEM models via the balanced truncation proper orthogonal decomposition," IEEE Trans. Antennas Propagat., vol. 59, no. 11, pp. 4142-4154, Nov. 2011. 
[6] X. Dang, M. Li, F. Yang, and S. Xu, "Quasi-periodic array modeling using reduced basis method," IEEE Antennas Wireless Propagat. Lett. vol. 16, pp. 825-828, 2016

[7] M. Rewienski, A. Lamecki, and M. Mrozowski, "Greedy multipoint model-order reduction technique for fast computation of scattering parameters of electromagnetic systems," IEEE Trans. Microwave Theory Tech., vol. 64, no. 6, pp. 1681-1693, Jun. 2016.

[8] G. Fotyga, M. Czarniewska, A. Lamecki, and M. Mrozowski, "Reliable greedy multipoint model-order reduction techniques for finite-element analysis," IEEE Antennas Wireless Propagat. Lett., vol. 17, no. 5, pp. $821-824,2018$.

[9] V. de la Rubia and J. Zapata, "An efficient method for determining TE and TM modes in closed waveguides made up of $N$ cylindrical conductors," IEEE Trans. Microwave Theory Tech., vol, 53, no. 2, pp. 670-678, Feb. 2005

[10] P. Kowalczyk, "Complex root finding algorithm based on delaunay triangulation," ACM Trans. Math. Softw., vol, 41, no. 3, p. 19, 2015.

[11] — " "On root finding algorithms for complex functions with branch cuts," J. Comput. Appl. Math., vol. 314, pp. 1-9, 2017.

[12] P. Kowalczyk and W. Marynowski, "Efficient complex root tracing algorithm for propagation and radiation problems," IEEE Trans. Antennas Propagat., vol. 65, no. 5, pp. 2540-2546, 2017.

[13] J. J. Michalski, "Complex border tracking algorithm for determining of complex zeros and poles and its applications," IEEE Trans. Microwave Theory Tech. vol. 66, no. 12, pp. 5383-5390, 2018

[14] P. Kozakowski and M. Mrozowski, "Quadratic programming approach to coupled resonator filter cad," IEEE Trans. Microwave Theory Tech., vol. 54, no. 11, pp. 3906-3913, Nov. 2006. 